\title{
REVERSIBLE MARKOV PROCESSES ON GENERAL SPACES AND SPATIAL MIGRATION PROCESSES
}

\author{
RICHARD F. SERFOZO, ${ }^{*}$ Georgia Institute of Technology
}

\begin{abstract}
In this study, we characterize the equilibrium behavior of spatial migration processes that represent population migrations, or birth-death processes, in general spaces. These processes are reversible Markov jump processes on measure spaces. As a precursor, we present fundamental properties of reversible Markov jump processes on general spaces. A major result is a canonical formula for the stationary distribution of a reversible process. This involves the characterization of two-way communication in transitions, using certain Radon-Nikodým derivatives. Other results concern a Kolmogorov criterion for reversibility, time reversibility, and several methods of constructing or identifying reversible processes.
\end{abstract}

Keywords: Reversible Markov process; Kolmogorov criterion; multiclass birth-death process; mobile phone; batch birth; batch death

2000 Mathematics Subject Classification: Primary 60J75

Secondary $60 \mathrm{~K} 20$

\section{Introduction}

In this study, we develop Markovian models of spatial migration, or particle systems whose dynamics are reversible. In doing this, we also extend several classical results for reversible Markov jump processes on countable spaces to the case of uncountable state spaces. This involves technicalities about communication in general spaces that are not issues in countable spaces.

The notion of reversibility of Markov processes was introduced by Kolmogorov [10]; see the review article [1]. An ergodic continuous-time (or discrete-time) Markov chain on a countable state space is reversible if, in equilibrium, the expected number of transitions per unit time from one state to another is equal to the expected number of transitions in the reverse direction. This is also equivalent to a time-reversibility property; namely that, at any instant, viewing the future of the process is stochastically indistinguishable from viewing the process in reverse time. More importantly, one can readily obtain the stationary distribution of a countable-state reversible Markov chain using the canonical formula (4), below, which is simply a product of ratios of the chain's transition rates (or transition probabilities).

The first part of our study is on fundamentals of reversible Markov jump processes on general state spaces (e.g. measure-valued processes and discrete-time Markov chains on general spaces). Section 2 contains preliminaries, including an example of multiclass birth-death processes. Section 3 contains a canonical formula for invariant measures (or stationary distributions)

Received 9 February 2005; revision received 24 April 2005.

* Postal address: School of Industrial and Systems Engineering, Georgia Institute of Technology, Atlanta, GA 30332, USA. Email address: rserfozo@isye.gatech.edu

Research supported in part by NSF grant DDM-9821123. 
of reversible Markov processes. This result involves characterizing two-way communication between any two of the chain's states, in a general state space, in terms of a Radon-Nikodým derivative involving the transition kernel. This derivative for a countable-state chain is the classical ratio of the transition rate between the states to the rate for the reverse transition. Our analysis also involves extending Kolmogorov's classical reversibility criterion to irreducible chains on general spaces. Although the material in Sections 2 and 3 applies to discrete-time Markov chains, we present it for continuous-time jump processes, which tend to have more applications.

The second part of our study focuses on applications. In Section 4, we describe stationary distributions for spatial migration and birth-death processes. These have potential applications in the modeling of mobile phone systems and related spatial queueing systems [4], [12], [15], [16]. Related countable-state reversible Markov network models, beginning with Kingman's [9] population process, can be found in [6], [18], and [16]. Other works on spatial birth-death processes are [11], [3], [7], [8], and [15].

In Section 5, we show how reversible processes arise from functions of reversible kernels and from interacting reversible processes. The results are useful for identifying reversible processes and modeling reversible systems. One proposition says that a process with multiple instantaneous reversible jumps is also reversible. This applies to birth-death or queueing systems with batch or synchronous movements.

\section{Preliminaries}

We will use the following notation throughout this study. Suppose that $X \equiv\left\{X_{t}: t \geq 0\right\}$ is a Markov jump process on a general measurable space $(\mathbb{E}, \mathcal{E})$ with transition rate kernel

$$
q(x, A)=\lim _{t \rightarrow 0} t^{-1} \mathrm{P}\left\{X_{t} \in A \mid X_{0}=x\right\}, \quad A \in \mathcal{E} .
$$

Assume that $q(x, \mathbb{E})$ is finite and positive, and that the process $X$ has no instantaneous or absorbing sets and is regular (i.e. the number of jumps it takes in any finite time interval is finite). We adopt the convention that $q(x,\{x\}) \equiv 0$, which means that fictitious jumps from $x$ back to itself are not considered. (Beware that some studies use $q$ as the infinitesimal generator, where $q(x,\{x\}) \equiv-q(x, \mathbb{E})$ : we will not use this convention.) Unless specified otherwise, all the processes (or kernels) we use herein will satisfy these assumptions. The construction of such a Markov jump process is discussed, for instance, in [5].

The process $X$ evolves as follows. Each sojourn time of the process $X$ in a state $x$ is exponentially distributed with rate $q(x, \mathbb{E})$. At the end of such a sojourn, the process jumps into a set $A$ with probability $P(x, A)=q(x, A) / q(x, \mathbb{E})$. The sequence of states visited by $X$ is a discrete-time Markov chain on $\mathbb{E}$ (called the embedded chain) with one-step transition probability kernel $P(x, A)$.

Definition 1. The transition rate kernel $q$ (or the Markov process $X$ ) is reversible with respect to a measure $\pi$ on $\mathbb{E}$ if

$$
\pi(\mathrm{d} x) q(x, \mathrm{~d} y)=\pi(\mathrm{d} y) q(y, \mathrm{~d} x) \quad \text { on } \mathbb{E}^{2} .
$$

The equality of measures (1), called detailed balance, implies the total balance

$$
\pi(\mathrm{d} x) q(x, \mathbb{E})=\int_{\mathbb{E}} \pi(\mathrm{d} y) q(y, \mathrm{~d} x) .
$$


Consequently, $\pi$ is an invariant measure for $q$. By Proposition 2, below, it is also an invariant measure for the transition probability of $X$. When $\pi$ is finite, it can be normalized to be the stationary probability measure of the process.

The preceding definition of reversibility and the results below for continuous-time processes also apply to discrete-time Markov chains - just interpret $q(x, A)$ in the results as a one-step transition probability kernel.

If $q$ is reversible with respect to $\pi$ then it follows, by induction on $n$, that, for $x_{0}, \ldots, x_{n} \in \mathbb{E}$,

$$
\pi\left(\mathrm{d} x_{0}\right) q\left(x_{0}, \mathrm{~d} x_{1}\right) \cdots q\left(x_{n-1}, \mathrm{~d} x_{n}\right)=\pi\left(\mathrm{d} x_{n}\right) q\left(x_{n}, \mathrm{~d} x_{n-1}\right) \cdots q\left(x_{1}, \mathrm{~d} x_{0}\right) \quad \text { on } \mathbb{E}^{n+1} .
$$

This equality of measures is the basis for the Kolmogorov criterion and the canonical form of invariant measures in the next section. Property (2) is also used to prove other properties, such as the fact that processes with batch or multiple instantaneous reversible transitions are reversible (Proposition 4).

The following results describe how the reversibility of $X$ is manifested in its embedded Markov chain, its transition probabilities, and its evolution in time.

Proposition 1. The rate kernel $q$ is reversible with respect to $\pi$ if and only if the transition probability kernel $P(x, A)$ for its embedded Markov chain is reversible with respect to $q(x, \mathbb{E}) \pi(\mathrm{d} x)$.

This result follows directly from the definition of reversibility. The next result is obvious for discrete-time Markov chains on countable state spaces, but it is a little more involved when there are exponential sojourn times. The memoryless property of a sojourn time acts like a local reversibility condition. Here and below, if $\pi$ is a measure on $\mathbb{E}$ and $q(x, \mathrm{~d} y)$ is a rate kernel, we let $\pi q$ denote the measure on the product space $\mathbb{E}^{2}$ defined by

$$
\pi q(\mathrm{~d} x \times \mathrm{d} y) \equiv \pi(\mathrm{d} x) q(x, \mathrm{~d} y) .
$$

Proposition 2. The rate kernel $q$ is reversible with respect to $\pi$ if and only if the transition probability kernel

$$
P_{t}(x, A):=\mathrm{P}\left\{X_{t} \in A \mid X_{0}=x\right\}
$$

is reversible with respect to $\pi$ for each $t$.

Proof. Suppose that $q$ is reversible with respect to $\pi$, and let $P_{t, n}(x, A)$ be the probability of $X$ starting in state $x$ and being in $A$ at time $t$, with this being the $n$th state visited by the process. We can then write

$$
P_{t}(x, A)=\sum_{n=0}^{\infty} P_{t, n}(x, A) .
$$

To prove that $P_{t}(x, A)$ is reversible with respect to $\pi$, it suffices to show that $P_{t, n}(x, A)$ is reversible with respect to $\pi$ for each $n \geq 0$. We will show this by induction on $n$.

The claim is true for $n=0$, since

$$
\pi P_{t, 0}(A \times B)=\int_{A \cap B} \mathrm{e}^{-q(x) t} \pi(\mathrm{d} x)=\pi P_{t, 0}(B \times A),
$$


where $q(x):=q(x, \mathbb{E})$. Now assume that the induction hypothesis is true for $n$. Then, conditioning on a jump out of state $x$, we have

$$
\begin{aligned}
\pi P_{t, n+1}(\mathrm{~d} x \times \mathrm{d} y) & =\int_{0}^{t} \pi(\mathrm{d} x) \int_{\mathbb{E}} q(x, \mathrm{~d} z) P_{t-s, n}(z, \mathrm{~d} y) \mathrm{e}^{-q(x) s} \mathrm{~d} s \\
& =\int_{0}^{t} \int_{\mathbb{E}} q(z, \mathrm{~d} x) \pi(\mathrm{d} z) P_{t-s, n}(z, \mathrm{~d} y) \mathrm{e}^{-q(x) s} \mathrm{~d} s \\
& =\pi(\mathrm{d} y) \int_{0}^{t} \int_{\mathbb{E}} q(z, \mathrm{~d} x) P_{t-s, n}(y, \mathrm{~d} z) \mathrm{e}^{-q(x) s} \mathrm{~d} s \\
& =\pi(\mathrm{d} y) \int_{0}^{t} \int_{\mathbb{E}} q(z, \mathrm{~d} x) P_{u, n}(y, \mathrm{~d} z) \mathrm{e}^{-q(x)(t-u)} \mathrm{d} u \\
& =\pi P_{t, n+1}(\mathrm{~d} y \times \mathrm{d} x) .
\end{aligned}
$$

The second equality follows by the reversibility of $q$, the third equality follows by the reversibility of $P_{t-s, n}$, and the fourth equality follows by the change of variable $u=t-s$. Consequently, $P_{t, n+1}$ is reversible with respect to $\pi$ and, hence, the induction is complete.

To prove the converse, suppose that $P_{t}$ is reversible with respect to $\pi$ for each $t$. From the well-known relation $P_{t}(x, A)=t q(x, A)+o(t)$, it follows that

$$
\begin{aligned}
\pi q(\mathrm{~d} x \times \mathrm{d} y) & =\lim _{t \downarrow 0} t^{-1} \pi P_{t}(\mathrm{~d} x \times \mathrm{d} y) \\
& =\lim _{t \downarrow 0} t^{-1} \pi P_{t}(\mathrm{~d} y \times \mathrm{d} x) \\
& =\pi q(\mathrm{~d} y \times \mathrm{d} x) .
\end{aligned}
$$

Thus, $q$ is reversible with respect to $\pi$.

The reversibility of $X$ is also related to it being 'reversible in time'. Specifically, for a fixed $\tau>0$, the time reversal of $X$ at $\tau$ is the process $X_{t}^{\tau}=X_{\tau-t}, 0 \leq t \leq \tau$. Each sample path of this process corresponds to a sample path of $X$ in reverse time starting at $\tau$ (like viewing a videotape in reverse). The process $X$ is reversible in time if, for each $\tau>0$, the process $X^{\tau}$ has the same distribution as $X$ on the interval $[0, \tau]$. In other words, the evolution of $X$ backward in time is equal in distribution to its evolution forward in time.

The time reversibility of $X$ is related to its reversibility as follows. Although this result is more technical than that for countable states, its proof is short since it uses Proposition 2, which is not needed in the case of countable states.

Proposition 3. The Markov process $X$ is reversible in time if and only if it is stationary and reversible.

Proof. Suppose that $X$ is reversible in time. Then each $X_{\tau}$ is equal in distribution to $X_{0}$, which implies that the Markov process $X$ is stationary. Let $\pi$ denote the distribution of $X_{0}$. Then, using stationarity and reversibility in time at $\tau=s+t$, we have

$$
\begin{aligned}
\pi(\mathrm{d} x) P_{t}(x, \mathrm{~d} y) & =\mathrm{P}\left\{\left(X_{s}, X_{s+t}\right) \in \mathrm{d} x \times \mathrm{d} y\right\} \\
& =\mathrm{P}\left\{\left(X_{s+t}, X_{s}\right) \in \mathrm{d} x \times \mathrm{d} y\right\} \\
& =\pi(\mathrm{d} y) P_{t}(y, \mathrm{~d} x) .
\end{aligned}
$$

This says that $P_{t}$ is reversible with respect to $\pi$ and, so, $q$ is also reversible with respect to $\pi$, by Proposition 2 . 
Conversely, suppose that $X$ is stationary and reversible, with stationary distribution $\pi$. Now $X$ will be reversible in time if, for any $0<t_{1}<\cdots<t_{n}<\tau$,

$$
\mathrm{P}\left\{X_{\tau-t_{1}} \in \mathrm{d} x_{1}, \ldots, X_{\tau-t_{n}} \in \mathrm{d} x_{n}\right\}=\mathrm{P}\left\{X_{t_{1}} \in \mathrm{d} x_{1}, \ldots, X_{t_{n}} \in \mathrm{d} x_{n}\right\} .
$$

Since $X$ is Markovian and stationary, this equality of measures is equivalent to

$$
\pi\left(\mathrm{d} x_{n}\right) \prod_{k=2}^{n} P_{t_{k}-t_{k-1}}\left(x_{k}, \mathrm{~d} x_{k-1}\right)=\pi\left(\mathrm{d} x_{1}\right) \prod_{k=2}^{n} P_{t_{k}-t_{k-1}}\left(x_{k-1}, \mathrm{~d} x_{k}\right) .
$$

However, this equality, which is analogous to (2), follows by the reversibility of $P_{t}$.

Quintessential examples of reversible processes are birth-death processes. Here are some variations on the classical birth-death process.

Example 1. (Up-down processes on partially ordered spaces.) Suppose that the process $X$ resides on a partially ordered space $\mathbb{E}\left(\right.$ e.g. $\mathbb{E}=\mathbb{R}^{m}$ ) with a measurable order relation ' $<$ '. Assume that its transition rate kernel is

$$
q(x, A)=\int_{A}\left[q_{1}(x, \mathrm{~d} y) \mathbf{1}(x<y)+q_{2}(x, \mathrm{~d} y) \mathbf{1}(y<x)\right],
$$

where $\mathbf{1}(\cdot)$ is an indicator function. When $X$ is in a state $x$, it may jump into a higher or lower set. Clearly, $X$ is reversible with respect to measures $\pi$ for which $\pi(\mathrm{d} x) q_{2}(x, \mathrm{~d} y)=$ $\pi(\mathrm{d} y) q_{1}(y, \mathrm{~d} x)$.

As an example, suppose that, on the space $\mathbb{E}=\mathbb{R}^{m}, X_{t}$ represents the state of $m$ continuous or discrete quantities at time $t$ (amounts of fluids at $m$ stations, outstanding orders at $m$ partsuppliers, stock levels of $m$ products in a warehouse, for example). Assume that the transition rate kernel of $X$ is

$$
q(\boldsymbol{x}, A)=\int_{A}\left[u(\boldsymbol{y}) u(\boldsymbol{x})^{-1} \mathbf{1}(\boldsymbol{x}<\boldsymbol{y})+v(\boldsymbol{x}) v(\boldsymbol{y})^{-1} \mathbf{1}(\boldsymbol{x}>\boldsymbol{y})\right] \psi(\mathrm{d} \boldsymbol{y}),
$$

where $u(\cdot)$ and $v(\cdot)$ are positive functions and $\psi$ is a measure on $\mathbb{E}$. Viewing $\tilde{u}(\boldsymbol{x}):=$ $-\log u(\boldsymbol{x})$ as a potential function, $u(\boldsymbol{y}) u(\boldsymbol{x})^{-1}=\mathrm{e}^{-(\tilde{u}(\boldsymbol{y})-\tilde{u}(\boldsymbol{x}))}$ reflects the change in potential by component 'increases' from $\boldsymbol{x}$ to $\boldsymbol{y}$. Similarly, $v(\boldsymbol{x}) v(\boldsymbol{y})^{-1}$ reflects a 'decrease' in a potential. In this case, $X$ is reversible with respect to $\pi(A)=\int_{A} u(\boldsymbol{x}) v(\boldsymbol{x})^{-1} \psi(\mathrm{d} \boldsymbol{x})$.

A special case of (3) is

$$
q(\boldsymbol{x}, A)=\int_{A} \exp \left\{-\sum_{j=1}^{m}\left[\alpha_{j}\left(x_{j}, y_{j}\right] \mathbf{1}\left(y_{j}>x_{j}\right)+\beta_{j}\left(y_{j}, x_{j}\right] \mathbf{1}\left(y_{j} \leq x_{j}\right)\right]\right\} \psi(\mathrm{d} \boldsymbol{y})
$$

where $\alpha_{j}$ and $\beta_{j}$ are measures on $\mathbb{R}_{+}$. Think of $\mathrm{e}^{-\alpha_{j}\left(x_{j}, y_{j}\right]} \psi(\mathrm{d} \boldsymbol{y})$ as the birth rate of size $y_{j}-x_{j}$ in component $j$, and $\mathrm{e}^{-\beta_{j}\left(y_{j}, x_{j}\right]} \psi(\mathrm{d} \boldsymbol{y})$ as the death rate of size $x_{j}-y_{j}$ in component $j$. These rates are compounded (multiplied) over the $m$ components to form the entire transition rate. Then $X$ is reversible with respect to

$$
\pi(A)=\int_{A} \exp \left\{-\sum_{j=1}^{m}\left(\alpha_{j}\left(0, x_{j}\right]-\beta_{j}\left(0, x_{j}\right]\right)\right\} \psi(\mathrm{d} \boldsymbol{x}) .
$$


A classical example of this process is an $m$-dimensional, nonnegative, integer-valued birthdeath process with transition rates

$$
q(\boldsymbol{x}, \boldsymbol{y})=\sum_{i=1}^{m}\left[\alpha_{i}\left(x_{i}\right) \mathbf{1}\left(\boldsymbol{x}+\boldsymbol{e}_{i}=\boldsymbol{y}\right)+\beta_{i}\left(x_{i}\right) \mathbf{1}\left(\boldsymbol{x}-\boldsymbol{e}_{i}=\boldsymbol{y}\right)\right] .
$$

Here $\boldsymbol{e}_{i}$ is the unit vector with a 1 in position $i$ and 0 s elsewhere, the $\alpha_{i}\left(x_{i}\right)$ and $\beta_{i}\left(x_{i}\right)$ are the birth and deaths rates in dimension $i$, and only one $x_{i}$ changes in a transition. An invariant measure for this process is

$$
\pi(\boldsymbol{x})=\prod_{i=1}^{m} \prod_{k=1}^{x_{i}} \frac{\alpha_{i}(k-1)}{\beta_{i}(k)}
$$

\section{Invariant measures}

For a countable-state Markov jump process, there is a well-known Kolmogorov criterion for it to be reversible, and this criterion yields a canonical representation for its invariant measures. In this section, we describe similar results for processes on general spaces. The novelty is that one-step communication between a pair of states of the process is characterized by certain Radon-Nikodým derivatives. This characterization is not needed in the case of countable states, where one-step communication is obviously characterized by a direct observation of the transition rate kernel.

To put our results into perspective, we first review the known canonical characterization of invariant measures for the Markov jump process $X$ when its state space $\mathbb{E}$ is countable. For simplicity, we write $q(x, y)=q(x,\{y\})$. Assume that $X$ has the two-way communication property that, for each $x \neq y \in \mathbb{E}$, the rates $q(x, y)$ and $q(y, x)$ are either both positive or both equal to 0 . We say that a sequence of states $x_{0}, x_{1}, \ldots, x_{n}$ in $\mathbb{E}$ is a path if $q\left(x_{i-1}, x_{i}\right)>0$, $i=1, \ldots, n$, and $n+1 \geq 2$. We also define the ratio of rates $\rho(x, y):=q(x, y) / q(y, x)$ for a path $x, y$.

The following well-known result characterizes invariant measures for reversible processes on countable state spaces (see, e.g. [6], [16], [18]).

Theorem 1. The following statements are equivalent.

(i) The transition rate function $q$ is reversible.

(ii) (Kolmogorov criterion.) For each $n \geq 3$ and $x_{1}, \ldots, x_{n}$ in $\mathbb{E}$ with $x_{n}=x_{1}$, we have

$$
\prod_{i=1}^{n-1} q\left(x_{i}, x_{i+1}\right)=\prod_{i=1}^{n-1} q\left(x_{i+1}, x_{i}\right)
$$

(iii) For each path $x_{0}, x_{1}, \ldots, x_{n}$ in $\mathbb{E}$ with $n+1 \geq 2$, the product $\prod_{i=1}^{n} \rho\left(x_{i-1}, x_{i}\right)$ (in general a function of $x_{0}, \ldots, x_{n}$, and $\left.n\right)$ is only a function of $x_{0}$ and $x_{n}$.

If any one of the preceding statements holds then $X$ is reversible and an invariant measure for it is

$$
\pi(x)=\prod_{i=1}^{n} \rho\left(x_{i-1}, x_{i}\right), \quad x \in \mathbb{E} \backslash\left\{x_{0}\right\}, \quad \pi\left(x_{0}\right)=1,
$$

where $x_{0}$ is an arbitrary state viewed as an origin and $x_{0}, \ldots, x_{n}=x$ is any path from $x_{0}$ to $x_{n}$. 
We will now prove an analogue of Theorem 1 for general state spaces. We will use the following shorthand notation. A measure $\mu$ on $\mathbb{E}^{2}$ is symmetric if $\bar{\mu}=\mu$, where $\bar{\mu}(\mathrm{d} x \times \mathrm{d} y) \equiv$ $\mu(\mathrm{d} y \times \mathrm{d} x)$ is the reversal of $\mu$. Recall that, for a measure $\pi$ on $\mathbb{E}$, we define the measure $\pi q$ on the product space $\mathbb{E}^{2}$ by $\pi q(\mathrm{~d} x \times \mathrm{d} y) \equiv \pi(\mathrm{d} x) q(x, \mathrm{~d} y)$. Then $q$ is reversible with respect to $\pi$ if and only if $\pi q$ is symmetric.

Our first task is to define two-way communication with the use of the following result of [17].

Lemma 1. For any $\sigma$-finite measure $\mu$ on $\mathbb{E}^{2}$, there is a symmetric set $\mathcal{R}_{\mu} \in \mathcal{E}^{2}$ such that $\mu$ on $\mathcal{R}_{\mu}$ is symmetric, and the Radon-Nikodým derivative $\mu(\mathrm{d} x \times \mathrm{d} y) / \bar{\mu}(\mathrm{d} x \times \mathrm{d} y)$ is positive $\mu$-almost everywhere ( $\mu$-a.e.) on $\mathcal{R}_{\mu}$. Furthermore, $\mu$ and $\bar{\mu}$ are orthogonal on $\mathcal{R}_{\mu}^{\mathrm{c}}$ (the complement of $\mathcal{R}_{\mu}$ ).

Consider the Markov jump process $X$, as in the preceding sections. We will characterize invariant measures for $X$ that are $\sigma$-finite. Some preliminaries are in order about its communication structure. Suppose, for now, that $X$ is reversible with respect to a $\sigma$-finite measure $\psi$ on $\mathbb{E}$ such that $\psi q$ is $\sigma$-finite. By applying Lemma 1 to the measure $\mu=\psi q$, we see that the Radon-Nikodým derivative

$$
\rho_{\psi}(x, y) \equiv \frac{\psi q(\mathrm{~d} x \times \mathrm{d} y)}{\overline{\psi q}(\mathrm{~d} x \times \mathrm{d} y)}
$$

is positive for $\psi q$-a.e. $(x, y)$ in $\mathcal{R}_{1}(\psi)=: \mathcal{R}_{\psi q}$. To avoid repeating the last phrase, we will assume that $\rho_{\psi}(x, y)>0$ for each $(x, y) \in \mathcal{R}_{1}(\psi)$. In addition, the measures $\psi q$ and $\overline{\psi q}$ vanish on $\mathcal{R}_{1}(\psi)^{\mathrm{c}}$, since they are equivalent and orthogonal on $\mathcal{R}_{1}(\psi)^{\mathrm{c}}$.

From these observations, the elements in the set $\mathcal{R}_{1}(\psi)$ can be viewed as the pairs of states that may communicate with each other in one step. Indeed, if $(x, y) \in \mathcal{R}_{1}(\psi)^{\mathrm{c}}$ then $y$ cannot be reached from $x$ in one step, and vice versa. On the other hand, if $(x, y) \in \mathcal{R}_{1}(\psi)$ then $y$ might be reached from $x$ in one step, and vice versa. However, the probability of a jump from $x$ to the singleton set $\{y\}$ is $q(x,\{y\}) / q(x, \mathbb{E})$, which may be 0 . Because of this property, the phrase 'may communicate' pertains to 'communication via derivatives' of transition rates, which differs from the usual notion of communication for a Markov process.

Note that if the state space $\mathbb{E}$ is countable and $\psi$ is a counting measure, then the derivative $\rho_{\psi}(x, y)$ equals the ratio $\rho(x, y)=q(x, y) / q(y, x)$ in Theorem 1 . It turns out that, for general state spaces, $\rho_{\psi}(x, y)$ plays a role similar to that of $\rho(x, y)$ for countable state spaces. The preceding observations justify the following terminology.

Definition 2. The set of routes of length $n \geq 2$ for the process $X$ is

$$
\mathcal{R}_{n}(\psi):=\left\{\left(x_{1}, \ldots, x_{n}\right) \in \mathbb{E}^{n}:\left(x_{i-1}, x_{i}\right) \in \mathcal{R}_{1}(\psi), 2 \leq i \leq n\right\} .
$$

The rate kernel $q$ has two-way $\psi$-communication on $\mathbb{E}$ if the following conditions are satisfied.

(a) The measure $\psi$ is $\sigma$-finite such that $\psi q$ is $\sigma$-finite and

$$
\sup _{(x, y) \in \mathcal{R}_{1}(\psi)} \rho_{\psi}(x, y)<\infty .
$$

(b) The measures $\psi q$ and $\overline{\psi q}$ vanish on $\mathcal{R}_{1}(\psi)^{\mathrm{c}}$.

(c) For $x, y \in \mathbb{E}$, there exist $x_{1}, \ldots, x_{n} \in \mathbb{E}$ such that $\left(x, x_{1}, \ldots, x_{n}, y\right) \in \mathcal{R}_{n+2}(\psi)$.

Condition (a) ensures that $X$ has a $\sigma$-finite invariant measure. Condition (b) is necessary for reversibility. If condition (c) were not to hold, there would be states that could not communicate 
with each other in a finite number of steps (or within a finite time), and we could delete these from the state space $\mathbb{E}$.

The following analogue of Theorem 1 characterizes invariant measures for reversible processes on general state spaces.

Theorem 2. Suppose that $q$ has two-way $\psi$-communication and define

$$
\rho_{\psi}\left(x_{1}, \ldots, x_{n}\right) \equiv \prod_{i=1}^{n-1} \rho_{\psi}\left(x_{i}, x_{i+1}\right), \quad\left(x_{1}, \ldots, x_{n}\right) \in \mathcal{R}_{n}(\psi) .
$$

The following statements are equivalent.

(i) The transition rate kernel $q$ is reversible with respect to a $\sigma$-finite measure that is equivalent to $\psi$.

(ii) $\rho_{\psi}\left(x_{1}, \ldots, x_{n}, x_{1}\right)=1$ for any $\left(x_{1}, \ldots, x_{n}, x_{1}\right) \in \mathcal{R}_{n+1}(\psi)$ and any $n \geq 2$.

(iii) For any $\left(x_{0}, \ldots, x_{n}, x\right) \in \mathcal{R}_{n+2}(\psi)$ and $n \geq 1$, the function $\rho_{\psi}\left(x_{0}, \ldots, x_{n}, x\right)$ depends only on $x_{0}$ and $x$.

If these statements hold then $X$ is reversible with respect to

$$
\pi(\mathrm{d} x)=f(x) \psi(\mathrm{d} x),
$$

where $f\left(x_{0}\right)=1$, for a fixed $x_{0}$ viewed as an origin, and

$$
f(x):=\rho_{\psi}\left(x_{0}, x_{1}, \ldots, x_{n}, x\right), \quad x \in \mathbb{E} \backslash\left\{x_{0}\right\},
$$

for any $\left(x_{0}, x_{1}, \ldots, x_{n}, x\right) \in \mathcal{R}_{n+2}(\psi)$.

Remark 1. (Construction of the function $f$.) Fix $x_{0} \in \mathbb{E}$ and let $\mathbb{E}_{0}=\left\{x_{0}\right\}$ and

$$
\mathbb{E}_{n+1}=\left\{x \in \mathbb{E} \backslash \bigcup_{k=0}^{n} \mathbb{E}_{k}:\left(x^{\prime}, x\right) \in \mathcal{R}_{1}(\psi) \text { for some } x^{\prime} \in \mathbb{E}_{n}\right\}, \quad n \geq 0 .
$$

Now define $f$ inductively on these disjoint sets according to $f\left(x_{0}\right)=1$ and

$$
f(x):=f\left(x^{\prime}\right) \rho_{\psi}\left(x^{\prime}, x\right), \quad x \in \mathbb{E}_{n}, \quad \text { for any } x^{\prime} \in \mathbb{E}_{n-1} .
$$

Note that $\bigcup_{n=1}^{\infty} \mathbb{E}_{n}=\mathbb{E}$ and that if $\mathbb{E}_{m}$ is empty, then $\mathbb{E}_{n}$ is empty for $n \geq m$.

Proof of Theorem 2. We first prove that part (i) implies part (ii). Suppose that $q$ is reversible with respect to a $\sigma$-finite measure $\pi$ which is equivalent to $\psi$. Let $r(x)$ denote a positive version of the Radon-Nikodým derivative $\pi(\mathrm{d} x) / \psi(\mathrm{d} x)$. The reversibility of $q$ implies the equality of measures

$$
r(x) \psi q(\mathrm{~d} x \times \mathrm{d} y)=r(y) \overline{\psi q}(\mathrm{~d} x \times \mathrm{d} y) .
$$

Then $\rho_{\psi}(x, y)=r(y) / r(x)$ in $\mathcal{R}_{1}(\psi)$. Using this in the definition (5) of $\rho_{\psi}$ and canceling terms yields part (ii).

We now prove that part (ii) implies part (iii). Assume that part (ii) is true. Fix $x_{0}$ and $x$, choose any routes, $\left(x_{0}, x_{1}, \ldots, x_{m}, x\right)$ in $\mathcal{R}_{m+2}(\psi)$ and $\left(x_{0}, y_{1}, \ldots, y_{n}, x\right)$ in $\mathcal{R}_{n+2}(\psi)$, from $x_{0}$ to $x$, and define

$$
a:=\rho_{\psi}\left(x_{0}, x_{1}, \ldots, x_{m}, x\right), \quad b:=\rho_{\psi}\left(x_{0}, y_{1}, \ldots, y_{n}, x\right) .
$$


To prove part (iii), it suffices to show that $a=b$. Define

$$
\bar{a}:=\rho_{\psi}\left(x, x_{m}, \ldots, x_{1}, x_{0}\right), \quad \bar{b}:=\rho_{\psi}\left(x, y_{n}, \ldots, y_{1}, x_{0}\right) .
$$

By the definition of $\rho_{\psi}$, we know that $\rho_{\psi}(x, y)=1 / \rho_{\psi}(y, x)$ and, so, $a=1 / \bar{a}$ and $b=1 / \bar{b}$. In addition, part (ii) implies that $a \bar{b}=b \bar{a}=1$. Consequently, $a=1 / \bar{a}=b$.

Finally, we prove that part (iii) implies part (i). Suppose that part (iii) holds. Then $f(x)$ defined by (7) is a function only of $x$, viewing $x_{0}$ as being fixed. The measure $\pi(\mathrm{d} x)=$ $f(x) \psi(\mathrm{d} x)$ is well defined and is $\sigma$-finite by property (a) of two-way communication (see Definition 2). Now, from the definitions of $\pi, \rho_{\psi}$, and $f$, we have

$$
\begin{aligned}
\pi q(\mathrm{~d} x \times \mathrm{d} y) & =f(x) \psi q(\mathrm{~d} x \times \mathrm{d} y) \\
& =f(x) \rho_{\psi}(x, y) \psi q(\mathrm{~d} y \times \mathrm{d} x) \mathbf{1}\left((x, y) \in \mathcal{R}_{1}(\psi)\right) \\
& =f(y) \psi q(\mathrm{~d} y \times \mathrm{d} x) \mathbf{1}\left((y, x) \in \mathcal{R}_{1}(\psi)\right) \\
& =\pi q(\mathrm{~d} y \times \mathrm{d} x) .
\end{aligned}
$$

Thus, $q$ is reversible with respect to $\pi$, which completes the proof.

Note that if the state space $\mathbb{E}$ is countable, then Theorem 2 (with $\psi(\{y\}) \equiv 1$ ) reduces to Theorem 1. The following corollary is another special case of Theorem 2.

Corollary 1. (Kernels with densities.) Suppose that the rate kernel is of the form

$$
q(x, \mathrm{~d} y)=r(x, y) \psi(\mathrm{d} y),
$$

where $r$ is a nonnegative function on $\mathbb{E}^{2}$. Then Theorem 2 holds with

$$
\rho_{\psi}(x, y)=\frac{r(x, y)}{r(y, x)},
$$

and the invariant measure in (6) is

$$
\pi(\mathrm{d} x)=\prod_{i=1}^{n} \rho_{\psi}\left(x_{i-1}, x_{i}\right) \psi(\mathrm{d} x),
$$

where $x_{n}=x$.

Here is an example of a process with a special communicating state.

Example 2. (Ōsawa [13], [14].) Suppose that, for the process $X$, there exists an $x_{0} \in \mathbb{E}$ such that

$$
q\left(x,\left\{x_{0}\right\}\right)>0 \text { for } q\left(x_{0}, \cdot\right) \text {-a.e. } x \in \mathbb{E}_{1}:=\mathbb{E} \backslash\left\{x_{0}\right\} .
$$

This means that, as the process $X$ moves within $\mathbb{E}_{1}$, it can reach $x_{0}$ in one jump from almost every state $x \in \mathbb{E}_{1}$. Therefore, $q$ is reversible with respect to a measure having $x_{0}$ as an atom if and only if

$$
q\left(x_{0}, \mathrm{~d} x\right) q(x, \mathrm{~d} y) q\left(y,\left\{x_{0}\right\}\right)=q\left(x_{0}, \mathrm{~d} y\right) q(y, \mathrm{~d} x) q\left(x,\left\{x_{0}\right\}\right) \quad \text { on } \mathbb{E}_{1}^{2} .
$$

In this case, $q$ is reversible with respect to a measure $\pi$ defined by $\pi\left(\left\{x_{0}\right\}\right)=1$ and

$$
\pi(\mathrm{d} x):=\frac{q\left(x_{0}, \mathrm{~d} x\right)}{q\left(x,\left\{x_{0}\right\}\right)} \quad \text { on } \mathbb{E}_{1}^{2} .
$$

This result is a special version of Theorem 2 with no $\sigma$-finiteness technicalities, where $f(x)=1$ in (6), and (8) is equivalent to $\rho_{\psi}(x, y)=1$ for $\pi$-a.e. $x$ and $y$. 


\section{Spatial migration and multiechelon processes}

In this section, we describe migrations of populations and birth-death systems that take place in a general space. The results also apply to abstract multiechelon processes.

Consider a system in which particles or items occasionally enter a space $S$ from outside and visit one or more locations in $S$ before departing. A typical state of the system is a finite counting measure $x$ on $S$ of the form $x(B)=\sum_{k=1}^{n} \delta_{s_{k}}(B)$, where $\delta_{s}(\cdot)$ is the Dirac measure with a unit atom at $s$. This $x(B)$ is the number of items in the set $B \in \&$, the $\sigma$-field on $S$. We let $x_{0}$ denote the zero measure on $S$. We model the system as a Markov process $X$ on the set $\mathbb{E}$ of finite counting measures on $S$, such that $X_{t}(B)$ denotes the number of items in the set $B$ at time $t$. The $\sigma$-field on $\mathbb{E}$ is the smallest one for which the function $x \mapsto x(B)$ is measurable for each $B \in \&$.

We assume that the rate of a transition of $X$ from state $x_{0}$ into $A \in \mathcal{E}$ is

$$
q\left(x_{0}, A\right)=\int_{S} \alpha\left(x_{0}, \mathrm{~d} s_{1}\right) \mathbf{1}\left(\delta_{s_{1}} \in A\right),
$$

and that, from any state $x=\sum_{i=1}^{n} \delta_{s_{i}}$, it has the three-action kernel

$$
\begin{aligned}
q(x, A)= & \int_{S} \alpha(x, \mathrm{~d} s) \mathbf{1}\left(x+\delta_{s} \in A\right)+\sum_{i=1}^{n} \beta\left(x, s_{i}\right) \mathbf{1}\left(x-\delta_{s_{i}} \in A\right) \\
& +\sum_{i=1}^{n} \int_{S} \lambda\left(x, s_{i}, \mathrm{~d} s\right) \mathbf{1}\left(x-\delta_{s_{i}}+\delta_{s} \in A\right) .
\end{aligned}
$$

Here, $\alpha(x, B)$ is the arrival rate kernel for an item entering a state in $B, \beta\left(x, s_{i}\right)>0$ is the departure rate for an item at $s_{i}$, and $\lambda\left(x, s_{i}, B\right)$ is the migration rate kernel for a one-step movement of an item from $s_{i}$ to another location in $B$. The process $X$ is a spatial migration process. We call it a spatial birth-death process when $\lambda(\cdot, \cdot, \cdot) \equiv 0$.

It is natural to express the state space as $\mathbb{E}=\bigcup_{n=0}^{\infty} \mathbb{E}_{n}$, where $\mathbb{E}_{0}=\left\{x_{0}\right\}$ and $\mathbb{E}_{n} \equiv$ $\{x: x(S)=n\}$, the set of counting measures of size $n$. According to the kernel (9), when $X$ is in a state $x \in \mathbb{E}_{n}$, it can only jump into a state in $\mathbb{E}_{n-1} \cup \mathbb{E}_{n} \cup \mathbb{E}_{n+1}$. Furthermore, from any $x \in \mathbb{E}_{n}$, the process can reach $x_{0}$ in $n$ transitions along a path in $\mathbb{E}_{n-1} \times \cdots \times \mathbb{E}_{0}$. In a slight abuse of notation, we let $\xi_{n}:=\sum_{i=1}^{n} \delta_{s_{i}}$, where $\xi_{0}=x_{0}$ (the zero measure).

For each $n \geq 1$, we define a measure $M_{n}$ on $S^{n}$ by

$$
M_{n}\left(\mathrm{~d} s_{n}\right) \equiv \prod_{k=1}^{n} \frac{\alpha\left(\xi_{k-1}, \mathrm{~d} s_{k}\right)}{\beta\left(\xi_{k}, s_{k}\right)},
$$

where $\boldsymbol{s}_{n}=\left(s_{1}, \ldots, s_{n}\right)$. The measure $M_{n}$ is symmetric if $M_{n}\left(\mathrm{~d} s_{n}\right)=M_{n}\left(\mathrm{~d} \tilde{\boldsymbol{s}}_{n}\right)$ on $S^{n}$ for any permutation $\tilde{s}_{n}$ of $s_{n}$.

Theorem 3. For the spatial migration process $X$, the following statements are equivalent.

(i) The process $X$ is reversible with respect to a measure that has an atom at $x_{0}$.

(ii) For any $\ell \geq 1$ and $x_{\ell}=x_{0}$, we have

$$
\prod_{k=1}^{\ell} q\left(x_{k-1}, \mathrm{~d} x_{k}\right)=\prod_{k=1}^{\ell} q\left(x_{k}, \mathrm{~d} x_{k-1}\right) \quad \text { on } \mathbb{E}^{\ell} .
$$


(iii) For each $n \geq 1$, the measure $M_{n}$ is symmetric and, for each $i$,

$$
M_{n}\left(\mathrm{~d} s_{n}\right) \lambda\left(\xi_{n}, s_{i}, \mathrm{~d} s\right)=M_{n}\left(\mathrm{~d}\left(s_{n}-\left(s_{i}-s\right) \boldsymbol{e}_{i}\right)\right) \lambda\left(\xi_{n}-\delta_{s_{i}}+\delta_{s}, s, \mathrm{~d} s_{i}\right) .
$$

When these statements hold, $X$ is reversible with respect to

$$
\pi:=\sum_{n=0}^{m} \pi_{n}
$$

where $\pi_{0}(A):=\mathbf{1}\left(x_{0} \in A\right)$ and, for $n \geq 1$,

$$
\pi_{n}(A):=\frac{1}{n !} \int_{S^{n}} M_{n}\left(\mathrm{~d} \boldsymbol{s}_{n}\right) \mathbf{1}\left(\xi_{n} \in A\right), \quad A \in \mathcal{E} .
$$

Proof. If part (i) holds then part (ii) follows by (2).

Assume that part (ii) holds. To prove that $M_{n}$ is symmetric, fix $s_{n} \in \mathbb{E}^{n}$ and let $\tilde{\boldsymbol{s}}_{n}$ be any permutation of it. Define $\xi_{k}:=\sum_{i=1}^{k} \delta_{s_{i}}, \tilde{\xi}_{k}:=\sum_{i=1}^{k} \delta_{\tilde{s}_{i}}$, and

$$
\left(x_{1}, \ldots, x_{2 n}\right):=\left(\xi_{1}, \ldots, \xi_{n}, \tilde{\xi}_{n-1}, \tilde{\xi}_{n-2}, \ldots, \tilde{\xi}_{0}\right) .
$$

For this sequence, (10) (with $\ell=2 n$ and $\xi_{0}=x_{0}=\tilde{\xi}_{0}$ ) is

$$
\prod_{k=1}^{n} q\left(x_{k-1}, \mathrm{~d} x_{k}\right) \prod_{j=0}^{n-1} q\left(x_{n+j}, \mathrm{~d} x_{n+j+1}\right)=\prod_{j=0}^{n-1} q\left(x_{n+j+1}, \mathrm{~d} x_{n+j}\right) \prod_{k=1}^{n} q\left(x_{k}, \mathrm{~d} x_{k-1}\right)
$$

that is,

$$
\prod_{k=1}^{n} \alpha\left(\xi_{k-1}, \mathrm{~d} s_{k}\right) \prod_{j=0}^{n-1} \beta\left(\tilde{\xi}_{n-j}, \tilde{s}_{n-j}\right)=\prod_{j=0}^{n-1} \alpha\left(\tilde{\xi}_{n-j-1}, \mathrm{~d} \tilde{s}_{n-j}\right) \prod_{k=1}^{n} \beta\left(\xi_{k}, s_{k-1}\right) .
$$

The second and fourth products are positive, since $\beta\left(\xi_{k}, s_{k}\right)>0$. Dividing this equality by these two products, and changing the indices $j$ to $k=n-j$, yields $M_{n}\left(\mathrm{~d} s_{n}\right)=M_{n}\left(\mathrm{~d} \tilde{\boldsymbol{s}}_{n}\right)$.

To prove (11), fix $s_{n} \in S^{n}$ and, for a fixed $i$ and $s$, define

$$
\boldsymbol{s}_{n}^{i}:=\left(s_{1}, \ldots, s_{i-1}, s, s_{i+1}, \ldots, s_{n}\right) .
$$

Let $\tilde{s}_{n}^{i}$ be any permutation of $s_{n}^{i}$, and define the measures $\xi_{k}^{i}=\sum_{j=1}^{k} \delta_{s_{j}}$, where $s_{i}=s$, and $\tilde{\xi}_{k}^{i}=\sum_{j=1}^{k} \delta_{\tilde{s}_{j}}$. Consider the sequence of states

$$
\left(x_{1}, \ldots, x_{2 n+1}\right):=\left(\xi_{1}, \ldots, \xi_{n}, \xi_{n}^{i}, \tilde{\xi}_{n-1}^{i}, \tilde{\xi}_{n-2}^{i}, \ldots, \tilde{\xi}_{0}^{i}\right)
$$

For this sequence, (10) is

$$
\begin{aligned}
& \prod_{k=1}^{n} \alpha\left(\xi_{k-1}, \mathrm{~d} s_{k}\right) \lambda\left(\xi_{n}^{i}, s_{i}, \mathrm{~d} s\right) \prod_{j=0}^{n-1} \beta\left(\tilde{\xi}_{n-j}^{i}, \tilde{s}_{n-j}\right) \\
& \quad=\prod_{k=1}^{n} \alpha\left(\tilde{\xi}_{k-1}^{i}, \mathrm{~d} \tilde{s}_{k}\right) \lambda\left(\xi_{n}^{i}, s, \mathrm{~d} s_{i}\right) \prod_{j=0}^{n-1} \beta\left(\xi_{n-j}, s_{n-j}\right) .
\end{aligned}
$$


By dividing both sides by the second and fourth products as we did above, we obtain (11). This completes the proof that part (ii) implies part (iii).

We end the proof by showing that part (iii) implies that $X$ is reversible with respect to $\pi$ as defined in (12). To show that $\pi q=\pi q$ on $\mathbb{E}^{2}$, it suffices, because of the structures of $\pi$ and $q$, to show that

$$
\begin{array}{ll}
\pi_{n} q=\overline{\pi_{n+1} q} & \text { on } \mathbb{E}_{n} \times \mathbb{E}_{n+1}, \text { for } n \geq 0, \\
\pi_{n} q=\overline{\pi_{n-1} q} & \text { on } \mathbb{E}_{n} \times \mathbb{E}_{n-1}, \text { for } n \geq 1, \\
\pi_{n} q=\overline{\pi_{n} q} & \text { on } \mathbb{E}_{n}^{2}, \text { for } n \geq 1 .
\end{array}
$$

Now, for any nonnegative (measurable) function $f$ on $\mathbb{E}_{n} \times \mathbb{E}_{n+1}$, it follows, by the definitions of $\pi_{n}, q$, and $\xi_{k}$, that

$$
\begin{aligned}
\pi_{n} q f & :=\int_{\mathbb{E}_{n} \times \mathbb{E}_{n+1}} \pi_{n} q(\mathrm{~d} x, \mathrm{~d} y) f(x, y) \\
& =\int_{S^{n}} \frac{M_{n}\left(\mathrm{~d} s_{n}\right)}{n !} \int_{S} \alpha\left(\xi_{n}, \mathrm{~d} s_{n+1}\right) f\left(\xi_{n}, \xi_{n+1}\right) .
\end{aligned}
$$

Then, by the definition and symmetry of $M_{n}$, we have

$$
\begin{aligned}
\pi_{n} q f & =\int_{S^{n+1}} \frac{M_{n+1}\left(\mathrm{~d} s_{n+1}\right)}{n !} \beta\left(\xi_{n+1}, s_{n+1}\right) f\left(\xi_{n}, \xi_{n+1}\right) \\
& =\sum_{i=1}^{n+1} \int_{S^{n+1}} \frac{M_{n+1}\left(\mathrm{~d} s_{n+1}\right)}{(n+1) !} \beta\left(\xi_{n+1}, s_{i}\right) f\left(\xi_{n+1}-\delta_{s_{i}}, \xi_{n+1}\right) \\
& =\int_{\mathbb{E}_{n+1} \times \mathbb{E}_{n}} \frac{}{\pi_{n+1} q}(\mathrm{~d} x, \mathrm{~d} y) f(x, y) .
\end{aligned}
$$

This proves the first line of (13). The second line follows by a similar argument.

To prove the third line of (13), let $f$ be a nonnegative function on $\mathbb{E}^{2}$. Then, using property (11) and letting $\eta_{n}:=\xi_{n}-\delta_{s_{i}}+\delta_{s}$, we have

$$
\begin{aligned}
\pi_{n} q f & =\int_{S^{n}} \frac{M_{n}\left(\mathrm{~d} s_{n}\right)}{n !} \sum_{i=1}^{n} \int_{S} \lambda\left(\xi_{n}, s_{i}, \mathrm{~d} s\right) f\left(\xi_{n}, \eta_{n}\right) \\
& =\int_{S^{n}} \frac{M_{n}\left(\mathrm{~d}\left(s_{n}-\left(s_{i}-s\right) \boldsymbol{e}_{i}\right)\right)}{n !} \sum_{i=1}^{n} \int_{S} \lambda\left(y_{n}, s, \mathrm{~d} s_{i}\right) f\left(\xi_{n}, \eta_{n}\right) \\
& =\overline{\pi_{n} q} f .
\end{aligned}
$$

This completes the proof.

Example 3. (Spatial birth-death process with interactions.) Recall that the process in Theorem 3 is a spatial birth-death process when $\lambda(\cdot, \cdot, \cdot) \equiv 0$. Consequently, Theorem 3 applies to this case with the minor change that statement (iii) does not include the condition (11). The stationary distribution is still given by (12), since $M_{n}$ does not depend on $\lambda$. Using another approach, Preston [15] established the existence of the stationary distribution of such processes without specifying its form.

We now describe another special case of Theorem 3. For the spatial migration process described above, suppose that the measures

$$
\alpha(x, A)=: \alpha(A), \quad \beta(x, A)=: \beta(A), \quad \lambda(x, s, A)=: \lambda(s, A)
$$


are independent of $x$, and that the measure $\gamma(\mathrm{d} s):=\beta(s)^{-1} \alpha(\mathrm{d} s)$ is finite. Extend $\lambda$ and $\gamma$ to $S \cup\{0\}$, where 0 represents the 'outside' of $S$, by letting

$$
\lambda(0, A)=\alpha(A), \quad \lambda(s, 0)=\beta(s), \quad \gamma(\{0\})=1-\gamma(S) .
$$

In this setting, items arrive at the system according to a space-time Poisson process with mean intensity $\alpha(\mathrm{d} s) \mathrm{d} t$ and, until it exits, each item moves independently in $S$ according to the Markov kernel $\lambda$.

Theorem 4. Under the preceding assumptions, the spatial migration process $X$ is reversible with respect to a measure that has an atom at $x_{0}$ if and only if the kernel $\lambda$ on $S \cup\{0\}$ is reversible with respect to $\gamma$. When $X$ is reversible, its stationary distribution $\pi$ is that of a Poisson process on $S$ with intensity measure $\gamma$; that is,

$$
\pi(A)=\mathrm{e}^{-\gamma(S)}\left[\mathbf{1}\left(x_{0} \in A\right)+\sum_{n=1}^{m} \frac{1}{n !} \int_{S^{n}} \prod_{k=1}^{n} \gamma\left(\mathrm{d} s_{k}\right) \mathbf{1}\left(\xi_{n} \in A\right)\right] .
$$

This distribution is also the limiting distribution of $X$ if and only if $\gamma(S)<\frac{1}{2}$.

Proof. Under the assumption that the measures (14) are independent of $x$, it follows that $M_{n}\left(\mathrm{~d} s_{n}\right)=\prod_{k=1}^{n} \gamma\left(\mathrm{d} s_{k}\right)$ in Theorem 3, meaning that statement (iii) there is clearly equivalent to $\lambda$ on $S \cup\left\{s_{0}\right\}$ being reversible with respect to $\gamma$. In this setting, the Poisson process distribution (15) is the stationary distribution when $X$ is reversible.

Since $X$ is Markovian, if its limiting distribution exists then it equals its stationary distribution. Clearly, $X$ is a regenerative process with regeneration point 0 and, so, by the key renewal theorem, its limiting distribution exists if and only if $\mathrm{E} \tau<\infty$, where $\tau$ is the time between two successive visits to 0 . It remains to show that

$$
\mathrm{E} \tau<\infty \Leftrightarrow \gamma(S)<\frac{1}{2} .
$$

We can interpret $\tau$ as the duration of a busy period in an $\mathrm{M} / \mathrm{G} / \infty$ service system having Poisson arrivals with rate $\lambda(0, S)$ and independent service times distributed in the same way as the time $T$ it takes for a Markov process, denoted by $Y_{t}$, with rate kernel $\lambda$ to travel from 0 back to 0 . For such a system, it is known (see, e.g. [2]) that

$$
\mathrm{E} \tau<\infty \Leftrightarrow \lambda(0, S) \mathrm{E} T<1 .
$$

To establish the last inequality, note that, since $Y_{t}$ has a stationary distribution $\gamma$, we know that $\gamma(\{0\})=1 / \lambda(0, S) \mathrm{E} T_{0}$, where $T_{0}$ is the time interval between two successive entrances to 0 . However, $T_{0}$ is also the time $T$ taken to travel from 0 to 0 plus a sojourn time at 0 . Therefore,

$$
\mathrm{E} T_{0}=\frac{1}{\lambda(0, S) \gamma(\{0\})}=\mathrm{E} T+\lambda(0, S)^{-1}
$$

Then, clearly, we have

$$
\begin{aligned}
\lambda(0, S) \mathrm{E} T=\gamma(\{0\})^{-1}-1<1 & \Leftrightarrow \gamma(\{0\})>\frac{1}{2} \\
& \Leftrightarrow \gamma(S)<\frac{1}{2} .
\end{aligned}
$$

These equivalences and (17) prove (16). 
Remark 2. When the spatial migration process described in Theorem 4 is stationary, one can show, as in [16, p. 262, Exercise 6], that the departure process is a space-time Poisson process with intensity $\alpha(\mathrm{d} s) \mathrm{d} t$.

Two important features of spatial migration processes, and the process in Example 2, are that, first, they can reach an origin state in a finite number of steps from any state and, second, the origin is an atom of the stationary distribution. A class of processes with these features is as follows.

Example 4. (Multiechelon processes.) Consider a process $X$ with an origin state $x_{0}$ and whose other states are generated by vectors with components in a measurable space $(S, \delta)$. Specifically, suppose that $g$ is a measurable function from $\bigcup_{n=1}^{m} S^{n}$ onto $\mathbb{E} \backslash\left\{x_{0}\right\}$ such that $g\left(s_{n}\right)=g\left(\tilde{\boldsymbol{s}}_{n}\right)$ if and only if $\tilde{\boldsymbol{s}}_{n}$ is a permutation of $\boldsymbol{s}_{n}$. That is, each $x=g\left(\boldsymbol{s}_{n}\right)$ is generated by the vector $\boldsymbol{s}_{n}$, viewed as a multiset (a set that may include multiple entries). Also, $\mathbb{E}=\sum_{n=0}^{m} \mathbb{E}_{n}$, where $\mathbb{E}_{n}=\left\{g\left(s_{n}\right): s_{n} \in S^{n}\right\}$.

Assume that a transition of the process $X$ from any $x$ in the $n$th echelon $\mathbb{E}^{n}$ into $A \in \mathcal{E}$ is triggered, in analogy to (9), by the following three actions.

- A new component $s_{n+1}$ is appended to $s_{n}$ with rate $\alpha(x, \mathrm{~d} s)$, resulting in a transition from $x \in \mathbb{E}_{n}$ to $g\left(s_{n+1}\right) \in \mathbb{E}_{n+1}$.

- A component $s_{i}$ is deleted from $s_{n}$ with rate $\beta\left(x, s_{i}\right)>0$, resulting in a transition from $x \in \mathbb{E}_{n}$ to $g\left(s_{n}-s_{i} \boldsymbol{e}_{i}\right)$ in $\mathbb{E}_{n-1}$.

- A component $s_{i}$ in $s_{n}$ is replaced by $s$ with rate $\lambda\left(x, s_{i}, \mathrm{~d} s\right)$, resulting in a transition from $x \in \mathbb{E}_{n}$ to $g\left(s_{n}-\left(s_{i}-s\right) \boldsymbol{e}_{i}\right)$ in $\mathbb{E}_{n}$.

This process is then of the form $X_{t}=g \circ h\left(\tilde{X}_{t}\right)$, where $\tilde{X}_{t}$ is a spatial migration process as above and $h: x \mapsto s$ is the measurable function that identifies the atoms of $x$. The equilibrium behavior of $X$ can therefore be characterized by the above results for $\tilde{X}_{t}$.

\section{Inheritance of reversibility}

We now show that a reversible transition rate kernel gives rise, via various functions of the kernel, to a host of reversible processes. The results in this section are useful in recognizing whether a process is reversible and for constructing reversible processes.

We begin by describing reversible processes in which each transition consists of multiple instantaneous transitions triggered by a reversible kernel. Let $\tilde{X}$ denote a Markov jump process on $\mathbb{E}$ with transition rate kernel $\tilde{q}(x, A)$. Consider a variation of this process in which each transition consists of up to $m$ transitions of $\tilde{X}$ occurring simultaneously, such that the transition rate kernel of this new process $X$ is

$$
q(x, A)=\sum_{n=1}^{m} q_{n}(x, A),
$$

where $m \leq \infty$ is fixed and

$$
q_{n}(x, A):=\int_{\mathbb{E}^{n-1}} \tilde{q}\left(x, \mathrm{~d} x_{1}\right) \tilde{q}\left(x_{1}, \mathrm{~d} x_{2}\right) \cdots \tilde{q}\left(x_{n-1}, A\right) .
$$

The compounding (or multiplication) of the rates of $n$ single jumps under $\tilde{q}$ represents a 'macro' transition rate for $n$ 'instantaneous jumps' of $\tilde{X}$. Note that $X$ is a random time transformation 
$X_{t}=\tilde{X}_{\tau_{t}}$ of the original $\tilde{q}$-process, where the random time process $\tau_{t}$ is piecewise linear (having slope 1), with random increments at instants when multiple instantaneous jumps occur. Each increment in the random time process $\tau_{t}$ is the sum of the exponential sojourn times of the $\tilde{q}$-process in those states associated with the multiple instantaneous jumps. The following result says that the process $X$ inherits its reversibility from the reversibility of $\tilde{q}$.

Proposition 4. (Multiple instantaneous jumps.) The process $X$ with rate kernel given by (18) is reversible with respect to $\pi$ if $\tilde{q}$ is reversible with respect to $\pi$.

Proof. Suppose that $\tilde{q}$ is reversible with respect to $\pi$. Then, by (2), we have $\pi q_{n}=\overline{\pi q_{n}}$ for each $n$, and, so, $\pi q=\sum_{n=1}^{m} \pi q_{n}=\sum_{n=1}^{m} \overline{\pi q_{n}}=\overline{\pi q}$. Thus, $X$ is reversible with respect to $\pi$.

Example 5. (Batch birth-death processes.) Suppose that $X$ is a batch birth-death process on $\mathbb{E}=\mathbb{R}^{m}$ in which a batch of $n$ births or a batch of $n$ deaths (each of a general size) may occur instantaneously according to the kernel (18), where $\tilde{q}$ is given by (3). Since $\tilde{q}$ is reversible with respect to $\pi(A)=\int_{A} u(\boldsymbol{x}) v(\boldsymbol{x})^{-1} \psi(\mathrm{d} \boldsymbol{x}), X$ is also reversible with respect to this measure.

The process $X$ described in Proposition 4 can be viewed as an $m$-action process, in that each of its transitions is triggered by $m$ actions with associated kernels $q_{1}, \ldots, q_{m}$. In this case, each $q_{n}$ has a special structure generated by the single kernel $\tilde{q}$. Is there an analogue of Proposition 4 for multiaction processes with general kernels $q_{n}$ ? Here is an example with an analogous proof.

Proposition 5. (Multiaction processes.) Suppose that the rate kernel of the process $X$ is $q=$ $\sum_{n=1}^{m} q_{n}$, where each $q_{n}$ is a rate kernel and $m \leq \infty$. Assume that there exists a measure $\pi$ on $\mathbb{E}$ and a permutation $k_{1}, \ldots, k_{m}$ of $1, \ldots, m$ such that $\pi q_{n}=\overline{\pi q_{k_{n}}}$ for each $n \leq m$. Then $q$ is reversible with respect to $\pi$.

In the rest of this section, we consider a Markov process $X$ with a compound transition rate kernel

$$
q(x, \mathrm{~d} y)=r(x, y) \tilde{q}(x, \mathrm{~d} y),
$$

where $r(x, y)$ is a nonnegative function on $\mathbb{E}^{2}$ and $\tilde{q}$ is a transition rate kernel for a process $\tilde{X}$ on $\tilde{\mathbb{E}} \supseteq \mathbb{E}$. The process $X$ can be viewed as a transformation of $\tilde{X}$, in that the state space and set of possible transitions of $X$ may be smaller than those of $\tilde{X}$, and the exponential sojourn rate $\tilde{q}(x, \mathbb{E})$ of $\tilde{X}$ in state $x$ becomes $\int_{\mathbb{E}} r(x, y) \tilde{q}(x, \mathrm{~d} y)$ for $X$.

The next two results address the issue of how the reversibility of $X$ is related to that of $\tilde{X}$. The first result is a special case of the second.

Proposition 6. For the transition rate kernel q given by (19), suppose that $r(x, y)$ is a symmetric function. Then $q$ is reversible with respect to $\pi$ on $\mathbb{E}$ if and only if $\tilde{q}$ is reversible with respect to $\pi$ on $\tilde{\mathbb{E}}$.

Proposition 7. Let $\tilde{\pi}$ be a measure on $\mathbb{E}$ and $f$ be a positive function on $\mathbb{E}$, both associated with the transition rate kernel $q$ given by (19). Then any two of the following statements imply the third:

(a) $\tilde{q}$ is reversible with respect to $\tilde{\pi}$;

(b) the function $f(x) r(x, y)$ is symmetric $\tilde{\pi} \tilde{q}$-a.e.;

(c) $q$ is reversible with respect to $\pi(\mathrm{d} x)=f(x) \tilde{\pi}(\mathrm{d} x)$. 
Proof. Clearly, parts (a) and (b) imply part (c), since

$$
\pi q(\mathrm{~d} x \times \mathrm{d} y)=f(x) r(x, y) \tilde{\pi} \tilde{q}(\mathrm{~d} x \times \mathrm{d} y)=\pi q(\mathrm{~d} y \times \mathrm{d} x) .
$$

Similarly, parts (b) and (c) imply part (a), since

$$
\tilde{\pi} \tilde{q}(\mathrm{~d} x \times \mathrm{d} y)=[f(x) r(x, y)]^{-1} \pi q(\mathrm{~d} x \times \mathrm{d} y)=\tilde{\pi} \tilde{q}(\mathrm{~d} y \times \mathrm{d} x),
$$

where $0^{-1}=1$, by convention. Finally parts (a) and (c) imply part (b), since

$$
\begin{aligned}
f(x) r(x, y) \tilde{\pi} \tilde{q}(\mathrm{~d} x \times \mathrm{d} y) & =\pi q(\mathrm{~d} x \times \mathrm{d} y) \\
& =\pi q(\mathrm{~d} y \times \mathrm{d} x) \\
& =f(y) r(y, x) \tilde{\pi} \tilde{q}(\mathrm{~d} y \times \mathrm{d} x) \\
& =f(y) r(y, x) \tilde{\pi} \tilde{q}(\mathrm{~d} x \times \mathrm{d} y) .
\end{aligned}
$$

We close with examples of the preceding propositions. The process $X$ with rate kernel (19) represents $\tilde{X}$ with its transitions restricted to a symmetric set $C \in \mathcal{E}^{2}$ if

$$
r(x, y)=\mathbf{1}((x, y) \in C) .
$$

These restrictions are equivalent to

$$
r(x, y)=\mathbf{1}(h(x, y) \leq b),
$$

where $h$ is a symmetric function and $b$ is a constant. In particular, $X$ is a truncation of $\tilde{X}$ to $\mathbb{E}$ when $C=\mathbb{E}^{2}$. Proposition 6 yields the following result.

Corollary 2. Suppose that $X$ represents $\tilde{X}$ with its transitions restricted to a symmetric set, or that $X$ is a truncation of $\tilde{X}$. If $\tilde{X}$ is reversible with respect to $\pi$ on $\tilde{\mathbb{E}}$, then $X$ is reversible with respect to $\pi$ restricted to $\mathbb{E}$.

For instance, if the reversible spatial migration process described in Theorem 4 is restricted to contain at most $m$ items, then it is a truncation of the process to the subset $\bigcup_{n=0}^{m} \mathbb{E}^{n} \subset \bigcup_{n=0}^{\infty} \mathbb{E}^{n}$. Hence, its invariant measures are simply restrictions to the smaller subset of those measures for the original process.

Example 6. (Restrictions on multidimensional processes.) There are many natural transition restrictions and truncations for processes with state space $\mathbb{E} \subseteq \mathbb{R}_{+}^{m}$. Here are a few illustrations of functions for which Corollary 2 applies.

- Taking $r(\boldsymbol{x}, \boldsymbol{y})=\mathbf{1}(\boldsymbol{x} \leq \boldsymbol{y})+\mathbf{1}(\boldsymbol{x} \geq \boldsymbol{y})$ restricts transitions to be either up or down (where the vector inequalities are component-wise).

- Suppose that $\tilde{X}$ represents a system that requires certain resources to sustain it. Specifically, whenever it is in a state $\boldsymbol{x}=\left(x_{1}, \ldots, x_{m}\right)$, let it require a quantity $a_{i j} x_{j}$ of a resource $i \in I$ for each component $j$, and let $b_{i}$ be the maximum amount of resource $i$ that is available. Then, setting $r(\boldsymbol{x}, \boldsymbol{y})=\mathbf{1}\left(\sum_{j} a_{i j} x_{j} \leq b_{i}, i \in I\right)$ constrains the system to not exceed the resources.

- Taking $r(\boldsymbol{x}, \boldsymbol{y})=\mathbf{1}\left(\max _{j}\left\{\left|y_{j}-x_{j}\right|\right\} \leq b\right)$ limits the difference in any component at a transition to not exceed $b$. 
Reversible processes arise in many contexts in which several independent reversible processes are linked together by certain interactions; see [16, Chapter 2]. Here is an example.

Example 7. (Independent spatial processes with interactions.) Consider a multiclass migration process $\tilde{X}_{t}=\left(\tilde{X}_{t}^{1}, \ldots, \tilde{X}_{t}^{m}\right)$ consisting of $m$ independent spatial, reversible migration processes, as in Theorem 4 , where the $m$ classes of item move within the same space $S$. The stationary distribution is the product measure $\prod_{k=1}^{m} \tilde{\pi}_{k}$ for the $m$ independent processes. Now, consider the modification of the system with the constraint that the total number of items in the space $S$ cannot exceed a limit $\ell$. The items that arrive when $\ell$ items are already in the system are turned away and never return. A feasible vector of measures must therefore satisfy

$$
\sum_{k=1}^{m} x^{k}(S) \leq \ell .
$$

In addition, assume that the level $\ell$ varies according to a nonnegative reversible process $L_{t}$ that is independent of $\tilde{X}$ and has stationary distribution $\pi_{L}(\cdot)$. Under this constraint, the independent processes $\tilde{X}_{t}^{i}$ and $L_{t}$ are linked together into dependent processes, resulting in a new process $Y_{t}=\left(L_{t}, X_{t}^{1}, \ldots, X_{t}^{m}\right)$ on the set $\mathbb{E}$ of vectors $\left(\ell, x_{1}, \ldots, x_{m}\right)$ that satisfy (20). Corollary 2 implies that $Y$ is reversible with respect to the product measure $\pi_{L}(A) \prod_{k=1}^{m} \tilde{\pi}_{k}\left(B_{k}\right)$ restricted to $\mathbb{E}$.

Example 8. (Jumps affected by a random environment.) Suppose that the rate kernel $\tilde{q}$ represents a system that is subject to a random environment which affects its jumps as follows. Whenever the system is in state $x$, a jump to a new state $y$ is allowed with probability $r(x, y)$ and is forbidden with probability $1-r(x, y)$. This jump modification is independent of everything else. The resulting system state over time is a Markov process with transition rate kernel (19). According to Proposition 7, if $\tilde{q}$ is reversible with respect to $\tilde{\pi}$ then $q$ is reversible if and only if there exists a function $f$ such that $f(x) r(x, y)$ is symmetric $\tilde{\pi} \tilde{q}$-a.e. In this case, $q$ is reversible with respect to $f(x) \tilde{\pi}$.

Example 9. (Kernels with densities.) Suppose that $q(x, \mathrm{~d} y)=r(x, y) \psi(\mathrm{d} y)$, as in Corollary 1 . Then it follows, by Proposition 7, that $q$ is reversible if and only if there exists a function $f$ such that $f(x) r(x, y)$ is symmetric for $\psi$-a.e. $x$ and $y$. In this case, $q$ is reversible with respect to $f(x) \psi(\mathrm{d} x)$.

\section{References}

[1] Dobrushin, R. L., Sukhov, Yu. M. And Fritts, I. (1988). A. N. Kolmogorov - founder of the theory of reversible Markov processes. Russian Math. Surveys 43, 157-182.

[2] Feller, W. (1971). An Introduction to Probability Theory and Its Applications, Vol 2, 2nd edn. John Wiley, New York.

[3] GLÖTZL, E. (1981). Time reversible and Gibbsian point processes. I. Markovian spatial birth and death processes on a general phase space. Math. Nachr. 102, 217-222.

[4] Huang, X. and Serfozo, R. F. (1999). Spatial queueing systems. Math. Operat. Res. 24, 865-886.

[5] Kallenberg, O. (1997). Foundations of Modern Probability. Springer, New York.

[6] Kelly, F. P. (1979). Reversibility and Stochastic Networks. John Wiley, London.

[7] Kendall, W. S. (1997). On some weighted Boolean models. In Advances in Theory and Applications of Random Sets, ed. D. Jeulin, World Scientific, Singapore, pp. 105-120.

[8] Kendall, W. S. ANd Møller, J. (2000). Perfect simulation using dominating processes on ordered spaces, with application to locally stable point processes. Adv. Appl. Prob. 32, 844-865.

[9] Kingman, J. F. C. (1969). Markov population processes. J. Appl. Prob. 6, 1-18.

[10] Kolmogorov, A. N. (1936). Zur theorie der Markoffschen ketten. Math. Ann. 112, 155-160. 
[11] Lopes Garcia, N. (1995). Birth and death processes as projections of higher-dimensional Poisson processes. Adv. Appl. Prob. 27, 911-930.

[12] MøLler, J. (2000). On the rate of convergence of spatial birth-and-death processes. Ann. Inst. Statist. Math. 41, 565-581.

[13] Ōsawa, H. (1985). Reversibility of Markov chains with applications to storage models. J. Appl. Prob. 22, 123-137.

[14] Ōsawa, H. (1988). Reversibility of first-order autoregressive processes. Stoch. Process. Appl. 28, 61-69.

[15] Preston, C. J. (1977). Spatial birth-and-death processes. Bull. Inst. Internat. Statist. 46, 371-391.

[16] Serfozo, R. F. (1999). Introduction to Stochastic Networks. Springer, New York.

[17] Tierney, L. (1998). A note on Metropolis-Hastings kernels for general state spaces. Ann. Appl. Prob. 8, 1-9.

[18] Whittle, P. (1986). Systems in Stochastic Equilibrium. John Wiley, New York. 